\title{
RECONHECIMENTO FACIAL E AUTISMO
}

\author{
FRANCISCO B. ASSUMPÇÃO JR*, MARIA HELENA SPROVIER/**, \\ EVELYN KUCZYNSKI***, VERA FARINHA****
}

\begin{abstract}
RESUMO - Através da apresentação de quatro figuras com expressões faciais diversas, avaliamos a capacidade de reconhecimento de crianças autistas, comparando-as com crianças de inteligência normal e adultos normais. A comparação dos resultados obtidos foi realizada através do teste do qui-quadrado. As diferenças observadas mostraram-se significativas, reforçando a idéia de que existe uma dificuldade da percepção das expressões faciais nas crianças autistas, o que interfere diretamente nos relacionamentos sociais.
\end{abstract}

PALAVRAS-CHAVE: autismo, reconhecimento facial, prosopagnosia.

\section{Facial recognizing and autism}

ABSTRACT - Through the presentation of four facial expressions' illustrations, we evaluate the capacity of autistic childrens recognition, comparing with normal intelligence children and adults. The comparison of results was accomplished through the qui-square test. The differences observed were significant, showing that a disturbance of the facial expressions' perception is present in autistic children, and that it interferes directly in the social relationships.

KEY WORDS - autism; facial recognizing; prosopagnosy.

O autismo é visto hoje como uma síndrome comportamental com etiologias múltiplas e evolução de um distúrbio do desenvolvimento ${ }^{1}$, caracterizada por déficit na interação social e no relacionamento com os outros, associado a alterações de linguagem e comportamento. Sua diferenciação dos quadros de deficiência mental é difícil, sendo realizada principalmente através da presença de comprometimento qualitativo no desenvolvimento das interações sociais e nas habilidades comunicacionais que, nas deficiências mentais, mesmo quando presente, não é a característica mais importante ${ }^{2}$. Seu surgimento ocorre antes dos 3 anos de idade, com prevalência estimada de 4 a 5/ 10 000 e predominância no sexo masculino (3 a 4 para 1), sendo relacionado a fatores pré-, peri- e pósnatais. Dessa maneira, diferentes quadros genéticos e neurológicos são descritos como a ele relacionados, conforme diversos estudos descrevem ${ }^{1,3-5}$, sendo as alterações cromossômicas descritas em altas percentagens por Steffemberg ${ }^{6}$, embora de natureza inespecífica e variada ${ }^{7}$.

Sob o ponto de vista do funcionamento mental, embora hipóteses afetivas tenham permeado sua conceituação, atualmente considera-se que crianças autistas apresentam comprometimento na capacidade de meta-representação. Assim, a ausência desta característica cognitiva específica

Estudo realizado no Serviço de Psiquiatria da Infância e da Adolescência (SEPIA) do Instituto de Psiquiatria (IPq) do Hospital das Clínicas (HC) da Faculdade de Medicina da Universidade de São Paulo (FMUSP): *LivreDocente em Psiquiatria pela FMUSP; Diretor Técnico do SEPIA-IPq-HC-FMUSP; **Doutora em Serviço Social, responsável pelo programa de Família e Desenvolvimento na Residência em Psiquiatria Infantil do IPQ-HCFMUSP; ***Pediatra, Psiquiatra da Infância e Adolescência, Pós-graduanda em Psiquiatria pelo Departamento de Psiquiatria da FMUSP; ****Psicóloga. Aceite: 6-agosto-1999.

Dr. Francisco B. Assumpção Jr. - Instituto de Psiquiátria, Hospital das Clínicas FMUSP - Av. Ovídio Pires de Campo s/n - 05403-900 São Paulo SP - Brasil. 
dificultaria a compreensão dos próprios estados mentais, bem como o dos outros ${ }^{8}$, uma vez que seria ela que permitiria as interações sociais que envolvem atribuir estados mentais a outrem, tais como crenças e desejos ${ }^{9}$. A expressão facial é um meio comunicacional importante, que permite a percepção de informações vitais que caracterizam estados e atributos mentais ${ }^{10}$. Sua identificação é realizada, não somente como uma identificação classificatória e generalizada, mas também como uma identificação particularizada. Dessa maneira, a identificação de objetos genéricos é mais simples que a de indivíduos específicos, ou seja, a habilidade de classificar é mais simples que a de identificar ${ }^{11}$, sendo a primeira condição básica para o estabelecimento da segunda. Assim, mesmo considerandose a presença da primeira, não necessariamente observaríamos a segunda.

As emoções são caracterizadas por seu aparecimento precoce e por sua expressão universal, inclusive através das expressões faciais. Assim, uma emoção prazerosa ou desprazerosa é determinada pelos estímulos internos e externos percebidos pela criança. Gradativamente, a criança constrói, então, um banco de memória com as representações perceptuais de suas diferentes emoções e como, uma vez expressas, seus pais respondem a elas. Assim, seu mundo de representações emocionais é construído e desenvolvido em função da mobilização da energia mental proporcionada pela experiência geradora de emoções, dos mecanismos de auto regulação e do estágio de adaptação social que permitirá a utilização dessas emoções para a comunicação.

Considerando-se que muitas crianças autistas conseguem perceber algumas categorias específicas de reconhecimento, embora não consigam identificar adequadamente os estados mentais mais sutis ${ }^{12}$, é que estruturamos o presente estudo. Assim, estabelecemos como objetivo a hipótese de que crianças autistas possuem menor capacidade de discriminar estados mentais através das expressões faciais do que crianças sem patologia psiquiátrica, e que essa dificuldade não se deve ao simples comprometimento da inteligência geral, uma vez que o reconhecimento das categorias que constituem a expressão facial seria a base, que nos leva a crer que a criança autista, embora reconheça os elementos que compõe aquela expressão facial, não apresenta condições de, ao juntá-los, atribuir a esta um significado específico.

\section{MÉTODO}

Foram selecionadas figuras que expressassem emoções humanas básicas.

As primeiras figuras, retiradas de um livro infantil, ao serem testadas com adultos normais para que se verificasse seu poder discriminatório, mostraram-se inadequadas, uma vez que permitiam o estabelecimento de uma sequência de ações que confundia os participantes, não permitindo a especificidade no reconhecimento e na concordância das emoções expressas.

Foram, então, selecionadas 9 figuras do jogo "Brincando com as Expressões" da Toyster, destinado a crianças acima de 8 anos de idade. Esse jogo foi escolhido uma vez que apresentava uma grande sequência de figuras que mostravam expressões faciais diferentes. Ao serem apresentadas a adultos normais, escolhidos aleatoriamente, somente 4 delas mostraram-se capazes de produzir concordância de respostas. Por essa razão, só essas 4 expressões - alegria (Fig 1), tristeza (Fig 2), raiva (Fig 3) e surpresa (Fig 4) foram utilizadas no decorrer do projeto.

Tendo-as já identificado 30 adultos normais, com índice de concordância elevado, as mesmas expressões foram apresentadas a 51 crianças com inteligência normal provenientes da Escola Estadual de $1^{\circ}$. Grau Prof. João Clímaco e a 30 crianças portadoras de autismo. O grupo de crianças normais foi escolhido aleatoriamente, tendo como critério de inclusão somente a idade, a qual os caracterizava em período de operações concretas e em condições de identificar as expressões faciais apresentadas.

O grupo de portadores de autismo foi colhido no ambulatório específico do "Projeto Distúrbios do Desenvolvimento" do Serviço de Psiquiatria da Infância e da Adolescência do Instituto de Psiquiatria do Hospital das Clínicas da Faculdade de Medicina da Universidade de São Paulo, considerando-se seu diagnóstico a partir dos critérios do DSM-IV ${ }^{2}$ e contato interpessoal que permitisse a compreensão das questões propostas. Os índices de acertos dos diferentes grupos foram comparados entre si utilizando-se o teste do qui-quadrado, visando-se verificar correlação e significância. 


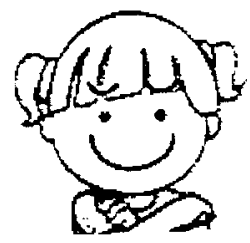

Fig.1

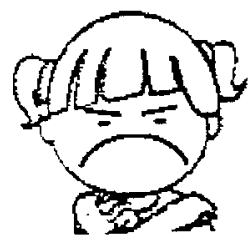

Fig.3

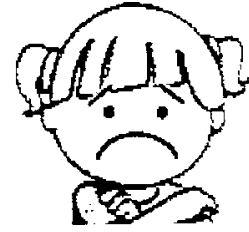

Fig..2

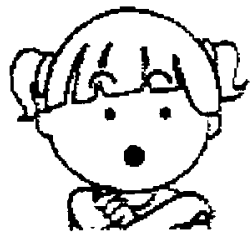

Fig.4

Figuras do Jogo "Brincando com as Expressões"- Toyster: 1. Alegria, 2. Tristeza;

3. Raiva; 4. Surpresa.

\section{RESULTADOS}

O grupo de adultos normais apresentou idade média de 22,66 $\pm 4,75$ anos; o de crianças normais, $8,98 \pm 2,75$ anos, e o de crianças autistas, 14,33 $\pm 5,23$ anos (QI=66 \pm 26$)$. Dessa maneira, o grupo de crianças autistas era composto basicamente por indivíduos com pequeno comprometimento intelectual e idade média mais avançada, o que nos permitiria pensar já terem condições de identificar corretamente o modelo apresentado, uma vez que, pelo seu nível de comprometimento intelectual, se encontrariam também em período de operações concretas.

Após a apresentação, na qual era pedido à criança que dissesse o que a menina da figura estava sentindo, obtivemos as respostas que constam desta tabelas (Tabelas 1 a 4).

Tabela 1. Comparação entre os grupos de adultos, crianças normais e crianças autistas quanto ao reconhecimento facial da expressão "alegre".

\begin{tabular}{lccc}
\hline & Crianças normais & Crianças autistas & Adultos normais \\
\hline Acertos & 48 & 13 & 30 \\
Erros & 03 & 17 & - \\
Total & 51 & 30 & 30 \\
\hline
\end{tabular}

Crianças normais $x$ crianças autistas: $\chi 2=26,198 ; p=0,0001$ (crianças normais e autistas diferem significativamente no reconhecimento da expressão "alegre")

Crianças normais $\mathrm{x}$ adultos normais: $\chi 2=1,833 ; \mathrm{p}=0,05$ (crianças e adultos normais não diferem significativamente no reconhecimento da expressão "alegre").

Crianças autistas $\mathrm{x}$ adultos normais: $\chi 2=23,721 ; \mathrm{p}=0,0001$ (crianças autistas diferem significativamente de adultos normais no reconhecimento da expressão "alegre"). 
Tabela 2. Comparação entre os grupos de adultos, crianças normais e crianças autistas quanto ao reconhecimento facial da expressão "triste".

\begin{tabular}{cccc}
\hline & Crianças normais & Crianças autistas & Adultos normais \\
\hline Acertos & 49 & 20 & 27 \\
Erros & 02 & 10 & 03 \\
Total & 51 & 30 & 30 \\
\hline
\end{tabular}

Crianças autistas $\mathrm{x}$ crianças normais: $\chi 2=12,948 ; \mathrm{p}=0,001 \quad$ (crianças autistas e normais diferem significativamente no reconhecimento da expressão "triste")

Crianças normais $\mathrm{x}$ adultos normais: $\chi 2=1,205 ; \mathrm{p}>0,05$ (crianças e adultos normais não diferem significativamente no reconheciemnto da expressão "triste")

Crianças autistas $\mathrm{x}$ adultos normais: $\chi 2=4,812$; $\mathrm{p}<0,05$ (diferem significativamente no reconhecimento da expressão "triste").

Tabela 3. Comparação entre os grupos de adultos, crianças normais e crianças autistas quanto ao reconhecimento facial da expressão "zangado".

\begin{tabular}{cccc}
\hline & Adultos & Crianças Normais & Crianças Autistas \\
\hline Acertos & 28 & 45 & 15 \\
Erros & 02 & 06 & 15 \\
Total & 30 & 51 & 30
\end{tabular}

Crianças autistas $\mathrm{x}$ crianças normais: $\chi 2=14,379 ; \mathrm{p}<0,0001$ (diferem significativamente no reconhecimento da expressão "zangado")

Crianças normais $\mathrm{x}$ adultos normais: $\chi 2=0,552 ; \mathrm{p}>0,05$ (não diferem no reconhecimento da expressão "zangado")

Crianças autistas $\mathrm{x}$ adultos normais: $\chi 2=8,422 ; \mathrm{p}<0,005$ (diferem significativamente no reconhecimento da expressão "zangado").

Tabela 4. Comparação entre os grupos de adultos, crianças normais e crianças autistas quanto ao reconhecimento facial da expressão "surpresa".

\begin{tabular}{cccc}
\hline & Adultos & Crianças Normais & Crianças Autistas \\
\hline Acertos & 26 & 24 & 08 \\
Erros & 04 & 27 & 22 \\
Total & 30 & 51 & 30
\end{tabular}

Crianças normais $\mathrm{x}$ crianças autistas: $\chi 2=3,841 ; \mathrm{p}>0,05$ (não diferem significativamente no reconhecimento da expressão "surpresa").

Crianças normais $\mathrm{x}$ adultos normais: $\chi 2=12,543 ; \mathrm{p}<0,0001$ (diferem significativamente no reconhecimento da expressão "surpresa").

Crianças autistas $\mathrm{x}$ adultos normais: $\chi 2=21,991 ; \mathrm{p}<0,001$ (diferem significativamente no reconhecimento da expressão "surpresa").

\section{DISCUSSÃO}

Em um diálogo, aquele que fala deve se colocar sempre a partir do ponto de vista daquele que escuta. $\mathrm{O}$ autista não é capaz de fazer isso. Mostra falta de empatia ou de habilidade para apreender o estado mental daquele que o escuta, não conseguindo um discurso comunicativo. Não obstante, o primeiro estudo que avaliou especificamente as habilidades pragmáticas do falar no autismo foi o de Baltaxe ${ }^{9}$. Nele, foram comparados os discursos de adolescentes autistas com o de crianças normais do estudo de Keenan \& Klein ${ }^{13}$, descobrindo-se que crianças autistas apresentam falhas frequentes na troca de papéis entre o que escuta e o que fala, e notando-se também que existe uma alteração dos princípios sociais da conversação, da educação e da cortesia. Assim, apesar de não quererem parecer 
rudes, parecem não compreender as regras sociais que governam uma conversação aceitável. Esses pacientes também não utilizam gestos com finalidade comunicativa. Assim, embora crianças autistas possam ter linguagem desenvolvida e utilizá-la intencionalmente (do modo como a utilizam com os objetos), isso não significa que se comuniquem de acordo com o conceito de comunicação, ou seja, de modo que quem fala afeta as intenções e crenças daquele que escuta. Utilizam a linguagem instrumental, embora não de maneira comunicativa.

É importante notar que o comportamento pragmático não se restringe somente à linguagem verbal. $\mathrm{O}$ uso de gestos com finalidade comunicativa também está comprometido nessas crianças ${ }^{14-16}$, da mesma forma que a compreensão e produção de expressões faciais comunicativas. Esse dado é visualizado em nosso estudo quando notamos diferenças, estatisticamente significativas, entre o grupo de crianças autistas e o de crianças normais, em que pese o fato de as crianças autistas apresentarem maior idade cronológica e nível intelectual compatível com deficiência mental leve.

Parece-nos, pela observação de nossos resultados, que a percepção das expressões faciais se estrutura de maneira diferente em cada uma delas, fato esse passível de observação na última expressão (de surpresa), na qual a amostra de acertos observados nas crianças normais era igual à das crianças autistas e estatisticamente diferente da dos adultos normais, levando-nos a supor que esse processo de reconhecimento se instale gradativamente para diferentes expressões. Isso também pode ser verificado quando comparamos o índice de acertos entre as diferentes expressões pelas crianças autistas, que mostram diferenças estatisticamente significativas entre os acertos das expressões $1 \mathrm{e}$ 2 (alegria e tristeza) e 2 e 4 (tristeza e surpresa), observando-se prejuízo mais acentuado na percepção da expressão da alegria e, principalmente de surpresa. Assim, parece-nos que o reconhecimento dessas expressões deva se produzir de maneira progressiva em relação ao desenvolvimento da criança e à maior complexidade da expressão apresentada. A literatura sobre a linguagem de crianças normais mostra uma competência pragmática precoce, uma vez que, ao redor de dois anos de idade, elas já podem adaptar a mensagem àquilo que a pessoa que escuta sabe ou não, e responder adequadamente às reações ou respostas daquele que ouve ${ }^{17,18}$. Para isso, a compreensão de algumas expressões faciais básicas faz-se necessária.

Crianças com atraso de linguagem ${ }^{19}$ e com síndrome de Down ${ }^{20}$ mostram atividade de linguagem com finalidade comunicativa, bem como adolescentes portadores de retardo mental ${ }^{21-23}$. Nas crianças e adolescentes portadores de autismo, essa finalidade comunicativa parece encontrarse prejudicada em todos os seus níveis, independentemente do retardo mental associado. Esse déficit na compreensão e na decodificação das manifestações emocionais do outro, estudadas neste trabalho a partir da não identificação da expressão facial, pode contribuir para o entendimento da dificuldade empática descrita usualmente nos portadores de autismo ${ }^{24}$. Entretanto, é importante percebemos que, além das dificuldades no reconhecimento facial, alterações em outros aspectos não verbais também estão presentes e, embora não possam ser considerados como característicos do quadro, uma vez que podem ocorrer em algumas outras variedades de retardo mental, podem ser vistas como a base do processo comunicativo e relacional falho ${ }^{25}$.

Uma teoria cognitiva de compreensão do autismo supõe que a capacidade para metarepresentações esteja comprometida nesta síndrome. Dessa maneira, a habilidade para mentalizar ou utilizar meta-representações (teoria da mente) não se manifesta desde o nascimento nem se adquire através da aprendizagem, mas se desenvolve de acordo com o crescimento da criança. Tal fato reforça nossos achados, fazendo-nos supor que a capacidade de decodificar as expressões faciais dependa de aspectos específicos de seu desenvolvimento, e não de seu desenvolvimento cognitivo global. Estudos reforçam esses nossos achados ${ }^{26}$, referindo que essa população não possui habilidade inata para o reconhecimento facial observada em crianças normais ou mesmo que sejam capazes de reproduzí-las, mesmo através do aprendizado, com uma dificuldade maior, principalmente na observação dos olhos. Essa última afirmação não corresponde ao encontrado por nós, uma vez que a maior dificuldade observada foi no reconhecimento da expressão da Figura 4, na qual a alteração 
mais significativa era o desenho da boca. Dois outros estudos ${ }^{27}$, que avaliam emoções através da expressão facial, encontraram comprometimento em amostra de pacientes autistas, no reconhecimento da expressão facial através de fotografias, embora esse déficit não fosse independente do comprometimento linguístico.

A teoria da mente caracterizaria, assim, um aspecto particular da inteligência, que permitiria a compreensão dos próprios estados mentais, bem como o dos outros, podendo ser o resultado de mudanças evolutivas a nível de córtex pré-frontal ${ }^{8}$ ou temporal ${ }^{28}$.

\section{REFERÊNCIAS}

1. Gillberg C. Infantile autism:diagnosis and treatment. Acta Psychiatr Scand 1990,81:209-215.

2. Apa. Manual de diagnóstico e estatística de transtornos mentais (DSM IV). São Paulo: Artes Médicas, 1995.

3. Coleman M. The autistic syndromes. Amsterdam: North Holland, 1976;183-208.

4. Finegan J, Quarrington B. Pre , peri and neonatal factors and infantile autism. J Child Psychol Psychiatry, 1979;20;119-128.

5. Rutter M. Psychological sequelae of brain damage in children. Am J Psychiatry, 1981:138;1533-1544.

6. Steffemberg S. Neuropsychiatric assessment of children with autism: a population-based study. Dev Med Child Neurol 1991;333;445-451.

7. Kuczynski E. Anormalidades cromossômicas esporádicas associadas à síndrome autística. Infanto Rev Neuropsiq Inf Adol 1996;4;26-36.

8. Povinelli DJ, Preuss TM. Theory of mind: evolutionary history of a cognitive specialization. Cortex $1995 ; 18 ; 418-424$.

9. Baltaxe C. Pragmatic deficits and the language of autistic adolescents. J Pediat Psychol 1976;2:176-180.

10. Davies S, Bishop D, Manstead ASR, Tantan D. Face perception in children with autism and Asperger's syndrome. J Child Psychol Psychiatry, 1994;35:1033-1057.

11. Ullman S. High-level vision: object recognition and visual cognition. Cambridge: MIT Press, 1997:159-168.

12. Baron-Cohen S. The development of a theory of mind in autism: deviance and delay? Psychiatr Clin North Am, 1991;14:33-51.

13. Keenan E, Klein E. Coherency in children's discourse. Psycholinguistic Res, 1975;4:365-380.

14. Bartak L, Rutter M, Cox A. A comparative study of infantile autism and specific developmental receptive disorder in children. Br J Psychiatry 1975;126-147.

15. Curcio F. Sensorimotor functioning and communication in autistic children. J Autism and Child Schizophr, 1978,8:281-292.

16. Ohta M. Cognitive disorders of infantile autism: a study employng the WISC, spatial relationship conceptualization and gestual imitations. J Autistic Dev Disord, 1987;17:45-52.

17. Furrow D. Social and private speech of years. Child Dev, 1984, 55:355-362.

18. Mueller C, Bleier M, Krakov J, Hegldus K, Carnoyer P. "The developmental of peak verbal interaction among 2-year-old boys." Child Dev, 1977;48:284-287.

19. Room A, Bliss S. A comparison of verbal comunication skills of language impaired and normal speaking children. $\mathrm{Br} \mathrm{J}$ Communic Disord, 1981;14:133-140.

20. Coggins TE, Carpenter RL, Owgins ND. "Examining early interpersonal demands. J Autism Dev Disord, 1983;11:201-217.

21. Bedrosian J. Communicative performance of mentally retarded adults in four conversational settings. J Speech Hear Res, 1978;21:79-95.

22. Longhurst TM. Communication in retarded adolescente and intelligence level. American J Mental Def; 1974;78:607-618.

23. Price-Williams D, Sabsay S. Communicative competence among severely retarded persons. Semiotica, 1979:26;35-63.

24. Attwood T. Asperger's syndrome. London: Jessica Kingsley, 1998:55-56.

25. Trevarthen C, Aitken K, Papondi D, Robarts J. Children with autism. London: Jessica Kingsley, 1997:34.

26. Peteers T. Autism. London: Whurr, 1977:119-121.

27. Rumsey JM. "Neuropsychological studies of high-level autism. In Schopler E, Mesibov GB.: High functioning individuals with autism. New York: Plenum, 1992:41-64.

28. Baron-Cohen S. Mindblindness. Cambridge: MIT Press, 1995:90-91. 